\title{
Comparison of Visual Outcomes of Ophthalmic Artery Aneurysms Treated with Microsurgical Clipping and Endovascular Coiling
}

\author{
Guangdong Lu, MD ${ }^{1, \star}$, Jaewoo Chung, $\mathrm{MD}^{2, \star}$, Jung Cheol Park, $\mathrm{MD}^{3}$, Jae Sung Ahn, $\mathrm{MD}^{3}$, \\ Byung Duk Kwun, $\mathrm{MD}^{4}$, Deok Hee Lee, $\mathrm{MD}^{5}$ \\ ${ }^{1}$ Department of Interventional Radiology, The First Affiliated Hospital of Nanjing Medical University, Nanjing, China \\ ${ }^{2}$ Department of Neurosurgery, Dankook University Hospital, Cheonan, Korea \\ ${ }^{3}$ Department of Neurosurgery, Asan Medical Center, University of Ulsan College of Medicine, Seoul, Korea \\ ${ }^{4}$ Department of Neurosurgery, Gangneung Asan Hospital, University of Ulsan College of Medicine, Gangneung, Korea \\ ${ }^{5}$ Department of Radiology, Research Institute of Radiology, Asan Medical Center, University of Ulsan College of Medicine, Seoul, Korea
}

Purpose: Post-treatment visual deficit is a major concern associated with both microsurgical clipping and endovascular coiling for the treatment of ophthalmic artery (OphA) aneurysms of the internal carotid artery. We aimed to compare the safety and effectiveness of the 2 modalities. Materials and Methods: We retrospectively reviewed and compared the baseline characteristics and postoperative visual and angiographic outcomes of OphA aneurysms treated by clipping or coiling between January 2010 and August 2018 at our hospital. In addition, the balloon occlusion test was performed to evaluate the safety of OphA occlusion.

Results: This study included 56 aneurysms treated by clipping and 82 aneurysms treated by coiling. Both the immediate and follow-up rates of incomplete aneurysm occlusion were comparable between the 2 groups (21.4\% vs. 22.0\%; $24.4 \%$ vs. 23.6\%). The incidence of post-treatment visual deficits was higher in the clipping group than in the coiling group (16.1\% vs. 2.4\%; $\mathrm{P}=0.010$ ). We observed total ipsilateral OphA occlusion in 6 patients and near occlusion in 3 patients during endovascular coiling; however, only 1 patient with near OphA occlusion showed a post-treatment visual field defect.

Conclusion: OphA aneurysms treated by endovascular coiling exhibited an aneurysm occlusion rate similar to that of microsurgical clipping with fewer post-treatment visual deficits. The total occlusion of OphA with adequate collaterals did not cause post-treatment visual deficits.

Key Words: Aneurysms; Balloon occlusion test; Clipping; Coiling; Ophthalmic artery; Vision, ocular

\section{INTRODUCTION}

Internal carotid artery (ICA) aneurysms at the origin of the ophthalmic artery (OphA), known as OphA aneurysms, are aneurysms arising at the junction between the ICA and the orifice of the OphA.' They are one of the most common ICA aneurysms related to the anterior clinoid process. ${ }^{2}$ Although both microsurgical clipping and endovascular treatment are effective for the management of OphA aneurysms, any visual complication could pose a major

\section{Correspondence to:}

Deok Hee Lee, MD

Department of Radiology, Research Institute of Radiology, Asan Medical Center, University of Ulsan College of Medicine, 88 Olympic-ro 43-gil, Songpa-gu, Seoul 05505, Korea

Tel: +82-2-3010-5944

Fax: +82-2-476-0090

E-mail:dhlee@amc.seoul.kr

*These authors contributed equally to the research work and preparation of the manuscript.

Received: May 17, 2020

Revised: January 2, 2022

Accepted: January 4, 2022
Copyright @ 2022 Korean Society of
Interventional Neuroradiology
This is an Open Access article distributed under the terms of
the Creative Commons Attribution Non-Commercial License
(http://creativecommons.org/licenses/by-nc/4.0) which
permits unrestricted non-commercial use, distribution, and
reproduction in any medium, provided the original work is
properly cited.
pISSN 2093-9043
eISSN 2233-6273 
concern for both strategies. Possible post-treatment visual deficits include reduction in visual acuity, visual field defects, or even blindness. ${ }^{2,3}$

Despite advances in endovascular technology, coiling for OphA aneurysms is still associated with an inherent risk of unintended thromboembolism or occlusion of the OphA itself, especially when the origin of the artery is adjacent to the neck or waist of the aneurysm sac. ${ }^{4-7}$ Although the balloon occlusion test (BOT) has been used to evaluate the sufficiency of the collateral supply to the OphA in case of arterial occlusion, 8,9 its usefulness during coiling for OphA aneurysms has not been well established. On the other hand, microsurgical clipping for OphA aneurysms is technically challenging due to the complex anatomy around the origin of OphA, owing to tight packing with critical structures including the ICA perforators, optic apparatus, oculomotor nerve, and cavernous sinus in this small region. ${ }^{10}$ Injury to these structures during exposure to the aneurysm neck may lead to visual deficits, bleeding, or stroke.

In addition, there is some concern associated with the treatment of OphA aneurysms using these 2 methods. It is often difficult to completely occlude OphA aneurysms while preserving the OphA during coiling if it has a wide neck incorporating the OphA itself. In contrast, the surgical clipping method offers relatively higher confidence in preserving the OphA. However, the possibility of a remnant aneurysm around the OphA still exists due to the poor visualization of the aneurysm from a narrow and complex anatomic location. $^{2}$

To the best of our knowledge, only a few studies have compared the use of microsurgical clipping and endovascular coiling for the treatment of OphA aneurysms. Therefore, in this report, we presented the results of OphA aneurysms treated in our hospital using these 2 methods and compared their safety and efficacy. We also attempted to determine whether occlusion of the OphA by violation of its orifice due to the use of coil loops would increase the incidence of visual deficits after endovascular coiling.

\section{MATERIALS AND METHODS}

\section{Study Population}

Data collection and analysis reported in this study were approved by the Institutional Review Board of our hospital. All patients diagnosed with OphA aneurysms using digital subtraction angiography (DSA) at our hospital between January 2010 and August 2018 were retrospectively reviewed. Aneurysms clearly associated with the OphA that could be clearly distinguished from other paraclinoid aneurysms were selected for this study. Only those patients who underwent treatment were included in the analysis and were divided either into the clipping group or the coiling group based on the treatment method. The treatment decision was made by each patient after discussion with neurointerventional physicians and neurosurgeons, based on their own characteristics. The data of patient characteristics, aneurysm features, procedural factors, aneurysm occlusion outcomes, post-treatment visual deficits, and clinical outcomes for the 2 groups were collected for comparison and analysis.

\section{Procedures}

Before the treatment, all aneurysms were evaluated by DSA in both groups, and a wide-neck aneurysm was defined as an aneurysm with a neck width $>4 \mathrm{~mm}$ or dome-to-neck ratio $<1.5$. The direction of the aneurysm was classified as superior, medial, or inferior based on the angular relationship between the aneurysm and the ICA as evaluated from the anterior projection on 3D rotational angiography. The relationship between the OphA and aneurysm was classified as "neck" or "sac" based on whether the OphA originated from the aneurysmal neck or was incorporated into the aneurysmal sac. ${ }^{8}$

\section{Balloon occlusion test}

The ICA BOT was selectively performed for aneurysms, of which the anticipated risk of OphA sacrifice was high when using clipping or coiling. A balloon catheter (Hyperglide; Medtronic, Irvine, CA, USA) was placed at the petrous or cavernous segment of the ipsilateral ICA through a 6-Fr guiding catheter in the common carotid artery (CCA). After ICA antegrade flow was completely occluded by inflating the balloon with contrast media, angiography was performed through the CCA to assess the collateral circulation between the external carotid artery (ECA) and OphA. Based on the BOT results, intact collateral circulation between the ECA and OphA was defined as retrograde filling of the OphA main trunk via ECA branches and development of retinochoroidal blush. ${ }^{8,9}$ The other cases were collectively referred to as poor collateral circulation. 


\section{Microsurgical clipping}

Microsurgical clipping was performed via the ipsilateral pterional approach by 2 experienced neurosurgeons. Patients were placed in the supine position with their heads facing the contralateral side. Intradural or extradural clinoidectomy was performed in cases whose paraclinoid aneurysm exposure was not proper in the surgical view. The falciform ligament and optic sheath were incised to expose the origin of the OphA and the aneurysm neck. The distal dural ring was incised around the ICA if the proximal neck of the aneurysm was not adequately exposed. Subsequently, the aneurysm was clipped with a single clip, or a combination of the appropriate straight, curved, angled, or fenestrated clips at the discretion of the surgeons. The OphA was preserved during the procedure, if possible. DSA was done on postoperative day 1 or 2 for the evaluation of the aneurysmal occlusion status and the OphA patency.

\section{Endovascular coiling}

Before the procedure, all patients were pre-medicated with dual antiplatelets (daily doses of aspirin $100 \mathrm{mg}$ and clopidogrel $75 \mathrm{mg}$ ) for at least 5 days without a loading dose. All procedures were performed using a biplane angiography system (Artis Zee; Siemens Healthcare, Forchheim, Germany) under general anesthesia. After introducing a 6-Fr guiding catheter (Envoy; Codman Neurovascular, Raynham, MA, USA) into the ICA on the same side of the aneurysm, a microcatheter was inserted and carefully advanced into the aneurysm under the roadmap guidance. The microcatheter tip was placed at the requisite position, after which coils of appropriate size were introduced. For wide-neck aneurysms with an unfavorable dome-neck ratio, the double microcatheter technique or the stent-assisted coiling technique was used. Intentional OphA occlusion was performed for selected aneurysms at the discretion of the operator but was performed only in patients who had adequate collateral supply from ECA during the BOT.

\section{Outcome Measurement}

Immediate post-procedural aneurysmal occlusion status and preservation of antegrade flow of the OphA were evaluated on the basis of the final angiogram recorded during the procedure in the coiling group and on the basis of the follow-up DSA, usually 1-2 days post-surgery, in the clipping group. Imaging follow-up by either DSA or computed tomographic angiography in the clipping group and by either DSA or magnetic resonance angiography in the coiling group was scheduled at 6-12 months post-surgery. The aneurysm occlusion status in the coiling group was evaluated by the Raymond-Roy classification. Raymond Class III in the coiling group and residual sac in the clipping group were regarded as incomplete occlusion of the aneurysm. Complete occlusion of OphA was defined as disappearance of previously antegrade blood flow, as seen on ipsilateral ICA angiography, while near OphA occlusion was defined as significantly slow yet antegrade blood flow.

Visual outcomes were carefully assessed for all patients before and after treatment. Visual deficit included any worsening of visual acuity, visual field defects, and blindness. In cases with pre-treatment visual deficit, the change of pre-treatment visual deficit was evaluated and classified into (1) improved and (2) unchanged. Post-treatment visual complications were defined as any newly developed visual deficit after the treatment.

Clinical outcomes were evaluated 1 month after the patient's discharge using the modified Rankin Scale (mRS) and were then classified as good (mRS score 0-2) or poor (mRS score 3-6).

\section{Statistical Analysis}

For convenient analysis, each patient with OphA aneurysms on both sides of the ICA was considered as 2 patients of the same age and sex but with OphA aneurysms on different sides. SPSS 24.0 (IBM Corp., Armonk, NY, USA) was used for all

Table 1. Characteristics of the 135 patients included in the current study

\begin{tabular}{lc}
\hline Patient characteristics & Value \\
\hline Age $(y)$ & $56.6 \pm 10.9(30-85)$ \\
Sex, female & $113(83.7)$ \\
Symptoms & $79(58.5)$ \\
Headache and/or dizziness & $16(11.9)$ \\
Preop visual deficit & $5(3.7)$ \\
Diplopia & $3(2.2)$ \\
Unilateral facial paresthesia & $41(30.4)$ \\
None & $37(27.4)$ \\
Hypertension & $32(23.7)$ \\
Other comorbidities & $49(36.3)$ \\
Multiplicity of aneurysms &
\end{tabular}

Values are presented as mean \pm standard deviation (range) or number (\%). 
statistical analyses, and the significance level for the analyses was fixed at 0.05. Quantitative variables were expressed as mean \pm standard deviation and were compared using an independent-sample t-test (normally distributed) or a ranksum test (abnormally distributed). Chi-square test or Fisher's exact test was performed to evaluate covariates for the binary categorical dependent variables. Logistic regression analysis using the forward stepwise method was used to evaluate the association between newly appeared visual deficits and suspected factors, and only factors with a P-value $<0.2$ in the univariate analysis were included in the multivariate analysis.

\section{RESULTS}

\section{Patient Characteristics}

In total, 267 OphA aneurysms in 264 patients were confirmed in our hospital between January 2010 and August 2018 using DSA. Among them, 56 aneurysms in 55 patients treated by microsurgical clipping and 82 aneurysms in 80 patients treated by coiling were included in the analysis. No aneurysm was ruptured. The baseline characteristics of the 135 patients are shown in Table 1.

The features of the patients and OphA aneurysms in the 2 groups are summarized in Table 2. There were no differences between the 2 groups in terms of baseline and aneurysm characteristics. Among the patients treated by coiling, a stent was used in 52 patients.

\section{Aneurysm Occlusion Outcomes}

Immediate incomplete aneurysm occlusion was confirmed in 12 (21.4\%) patients who underwent microsurgical clipping and in 18 (22.0\%) patients who underwent coiling. No difference was found between the 2 groups in terms of immedi-

Table 2. Features of patients and OphA aneurysms in the 2 patient groups and their treatment outcomes

\begin{tabular}{|c|c|c|c|}
\hline Variable & Clipping $(n=56)$ & Coiling $(n=82)$ & P-value \\
\hline Patient age (y) & $56.6 \pm 11.6$ & $56.5 \pm 10.3$ & 0.950 \\
\hline Sex, female & $48(85.7)$ & $67(81.7)$ & 0.535 \\
\hline Hypertension & $14(25.0)$ & $25(30.5)$ & 0.482 \\
\hline \multicolumn{4}{|l|}{ Aneurysm characteristics } \\
\hline Side, right & $22(39.3)$ & $41(50.0)$ & 0.215 \\
\hline Size $(\mathrm{mm})$ & $5.8 \pm 3.8(1.6-22)$ & $5.6 \pm 2.9(2.5-20)$ & 0.740 \\
\hline Lobulated or elongated aneurysms & $19(33.9)$ & $34(41.5)$ & 0.372 \\
\hline Aneurysm direction & & & 0.096 \\
\hline Superior & $32(57.1)$ & $35(42.7)$ & \\
\hline Medial & $17(30.4)$ & $25(30.5)$ & \\
\hline Inferior & $7(12.5)$ & $22(26.8)$ & \\
\hline Mean neck of aneurysms (mm) & $4.0 \pm 1.6$ & $3.9 \pm 1.5$ & 0.476 \\
\hline Wide-neck aneurysms ${ }^{\dagger}$ & $47(83.9)$ & $64(78.0)$ & 0.393 \\
\hline OphA originating from the sac & $34(60.7)$ & $50(61.0)$ & 0.975 \\
\hline Immediate incomplete obliteration & $12(21.4)$ & $18(22.0)$ & 0.942 \\
\hline Totally or nearly occluded OphA & $0(0)$ & $9(11.0)$ & $0.027^{*}$ \\
\hline Pre-treatment visual deficits & $9(16.1)$ & $7(8.5)$ & 0.175 \\
\hline Improved after treatment & $5(55.6)$ & $3(42.9)$ & 1.000 \\
\hline Unchanged deficits & $4(44.4)$ & $4(57.1)$ & 1.000 \\
\hline Post-treatment visual deficits & $9(16.1)$ & $2(2.4)$ & $0.010^{*}$ \\
\hline Clinical outcomes $(m R S>2)$ & $1(1.8)^{\ddagger}$ & $0(0)$ & 0.406 \\
\hline
\end{tabular}

Values are presented as mean \pm standard deviation (range) or number (\%).

OphA, ophthalmic artery; mRS, modified Rankin Scale.

* Statistically significant with $\mathrm{P}<0.05$. 'Wide-neck aneurysms were defined as aneurysms with a neck width $>4 \mathrm{~mm}$ or dome-to-neck ratio $<1.5$. ${ }^{\ddagger}$ Due to premature rupture of the previously non-ruptured aneurysm during clipping. 
ate incomplete occlusion ( $\mathrm{P}=0.942$ ). Imaging follow-up data were available for 45 (80.4\%) aneurysms treated by clipping and 55 (67.1\%) aneurysms treated by coiling. The median

Table 3. Test results of 51 patients who underwent the BOT in the 2 groups and their treatment outcomes

\begin{tabular}{lcc}
\hline Findigns & $\begin{array}{c}\text { Clipping } \\
(\mathrm{n}=12)\end{array}$ & $\begin{array}{c}\text { Coiling } \\
(\mathrm{n}=39)\end{array}$ \\
\hline Intact collateral circulation & $\mathrm{n}=9$ & $\mathrm{n}=34$ \\
Total occlusion of the OphA & 0 & $5(14.7)$ \\
Near occlusion of the OphA & 0 & $3(8.9)$ \\
Post-treatment visual deficit & $5(55.6)$ & $1(2.9)$ \\
Incomplete aneurysm occlusion & $3(33.3)$ & $7(20.6)$ \\
Poor collateral circulation & $\mathrm{n}=3$ & $\mathrm{n}=5$ \\
Total occlusion of the OphA & 0 & 0 \\
Near occlusion of the OphA & 0 & 0 \\
Post-treatment visual deficit & 0 & 0 \\
Incomplete aneurysm occlusion & $2(66.7)$ & $3(60.0)$ \\
\hline
\end{tabular}

Values are presented as number (\%).

OphA, ophthalmic artery; BOT, balloon occlusion test. follow-up time was 13.1 months in the clipping group and 19.0 months in the coiling group. At the latest follow-up, incomplete occlusion was found in $24.4 \%$ (11/45) of aneurysms treated by clipping and in 23.6\% (13/55) of aneurysms treated by coiling $(P=0.925)$. There was no incomplete occlusion among patients with total or near OphA occlusion in the coiling group.

\section{Visual Outcomes}

There were 16 patients ( 9 in the clipping group and 7 in the coiling group) with pre-treatment visual deficits. The 9 patients in the clipping group presented with decreased visual acuity in 6 (66.7\%) and visual field defect in 3 (33.3\%) before treatment. After clipping, the deficits improved in 5 (55.6\%) patients and were unchanged in 4 (44.4\%) patients. The 7 patients in the coiling group presented with decreased visual acuity in 5 (71.4\%) and visual field defect in 2 (28.6\%) before treatment. After coiling, the visual deficits improved in 3 (42.9\%) and were unchanged in 4 (57.1\%) patients. No patient showed aggravation of the pre-existing deficits in either

Table 4. Univariate analysis of post-treatment visual deficits in all included patients

\begin{tabular}{|c|c|c|c|}
\hline Variable & With visual deficits $(n=11)$ & Without visual deficits ( $n=127)$ & P-value \\
\hline Age (y) & $52.2 \pm 11.1$ & $56.9 \pm 10.8$ & 0.165 \\
\hline Sex, female & $11(100)$ & $106(83.5)$ & 0.304 \\
\hline Hypertension & $2(18.2)$ & $37(29.1)$ & 0.671 \\
\hline \multicolumn{4}{|c|}{ Aneurysm characteristics } \\
\hline Side, right & $3(27.3)$ & $60(47.2)$ & 0.202 \\
\hline Size $(\mathrm{mm})$ & $5.1(3.8-8.9)$ & $4.7(3.6-6.5)$ & 0.307 \\
\hline Irregular shape & $6(54.5)$ & $47(37.0)$ & 0.410 \\
\hline Direction & & & 0.193 \\
\hline Superior & $4(36.4)$ & $63(49.6)$ & \\
\hline Medial & $6(54.5)$ & $36(28.3)$ & \\
\hline Inferior & $1(9.1)$ & $28(22.0)$ & \\
\hline Wide necked ${ }^{\dagger}$ & $10(90.9)$ & $102(80.3)$ & 0.645 \\
\hline OphA origin & & & 0.245 \\
\hline From the sac & $9(81.8)$ & $75(59.1)$ & \\
\hline From the neck & $2(18.2)$ & $52(40.9)$ & \\
\hline Treatment modalities & & & $0.010^{*}$ \\
\hline Clipping & $9(81.8)$ & $47(37.0)$ & \\
\hline Coil embolization & $2(18.2)$ & $80(63.0)$ & \\
\hline
\end{tabular}

Values are presented as mean \pm standard deviation, number (\%), or median (quartile).

OphA, ophthalmic artery.

* Statistically significant with $\mathrm{P}<0.05$. ${ }^{\dagger}$ Wide-neck aneurysms were defined as aneurysms with a neck width $>4$ mm or dome-to-neck ratio $<1.5$. 
group. There was no difference in the frequency of visual deficit improvement between the 2 groups $(P>0.05)$.

OphA patency was observed in all patients treated by microsurgical clipping. However, post-treatment visual deficits occurred in 9 patients (16.1\%) in this group, and the deficits included decreased visual acuity in 2, visual field defect in 4, both in 2, and monocular blindness in 1. All post-treatment visual deficits occurred in patients who had undergone anterior clinoidectomy $(n=50)$ during clipping.

In the coiling group, total occlusion of the ipsilateral OphA, either intentional or unintentional, was observed in 6 patients, and near occlusion was observed in 3 patients. Intact collateral circulation was confirmed by BOT in 8 of these patients before treatment, and BOT was not performed in the other patient. However, ECA angiography after coiling demonstrated intact collateral circulation in this patient, and no visual deficit was observed. Post-treatment visual deficits occurred in 2 patients (2.4\%) in the coiling group. A patient with both visual field defect and reduction in visual acuity was found to show near OphA occlusion on final angiography; however, BOT before coiling showed intact collateral circulation in this patient. The other patient with only a reduction in visual acuity was found to have a well-preserved OphA during the procedure; however, this patient complained of foggy vision after waking up from the anesthesia, and small infarctions were observed on the retina by fundus examination. This patient did not undergo the BOT before the procedure.

The test results of 51 patients who underwent BOT in the 2 groups and their treatment outcomes are shown in Table 3. There were no complications in all 51 patients who underwent BOT and intact collateral circulation was demonstrated in 43 (84.3\%) patients. Total occlusion of ipsilateral OphA was found in 5 patients with adequate collaterals during coiling, and they showed no post-treatment visual deficits. A patient with near occlusion of ipsilateral OphA experienced visual deficits after coiling, although intact collateral circulation was confirmed in this patient by the BOT. No delayed visual complications occurred in either group during follow-up. A significant difference in the frequency of post-treatment visual deficits was found between the 2 groups: $16.1 \%(9 / 56)$ in the clipping group and $2.4 \%(2 / 82)$ in the coiling group $(P=0.010)$. Univariate analysis for the incidence of post-treatment visual deficits in all included patients is shown in Table 4. Only microsurgical clipping exhibited a significant difference in the incidence of post-treatment visual deficits (odds ratio 7.660,
95\% confidence interval 1.587-36.962, $\mathrm{P}=0.011$ ) by logistic regression analysis (Table 5). Patients with newly developed visual deficits and their follow-up outcomes are presented in Table 6. During follow-up, the deficits disappeared completely in 2 patients, improved in 7 patients, and remained unchanged in 2 patients.

\section{Other Complications and Clinical Outcomes}

In the clipping group, premature rupture of a previously unruptured aneurysm occurred in 1 patient, but without neurological deficits after clipping. In the coiling group, 1 patient developed a retroperitoneal hematoma requiring surgery. A delayed ischemic complication involving monoparesis of the upper arm 10 days after discharge occurred in this group. On the clinical follow-up at 1 month post-hospital discharge, all patients, except 1 in the clipping group with pre-existing thalamic hemorrhage, showed excellent outcomes.

\section{DISCUSSION}

By comparing the occurrence of post-treatment visual deficits and aneurysm occlusion outcomes in 2 groups, we were able to observe that patients treated by coiling showed a much lower frequency of post-treatment visual deficits and a comparable aneurysm occlusion rate. We also found that total occlusion of an OphA with an adequate collateral supply, confirmed by the BOT, was not associated with the incidence of post-treatment visual deficits.

\section{Visual Complications}

The reported incidence of post-treatment visual complica-

Table 5. Logistic regression analysis of post-treatment visual deficits

\begin{tabular}{lccc}
\hline Variable & $\begin{array}{c}\text { Odds } \\
\text { ratio }\end{array}$ & $\begin{array}{c}95 \% \text { confidence } \\
\text { interval }\end{array}$ & P-value \\
\hline Age & 0.962 & $0.908-1.019$ & 0.191 \\
$\begin{array}{l}\text { Direction } \\
\text { Superior }\end{array}$ & 1 & & 0.232 \\
$\quad$ Medial & 3.242 & $0.791-13.295$ & 0.102 \\
$\quad$ Inferior & 1.138 & $0.110-11.773$ & 0.914 \\
Treatment modalities & & & \\
$\quad$ Coil embolization & 1 & & \\
$\quad$ Clipping & 7.660 & $1.587-36.962$ & 0.011 \\
\hline
\end{tabular}




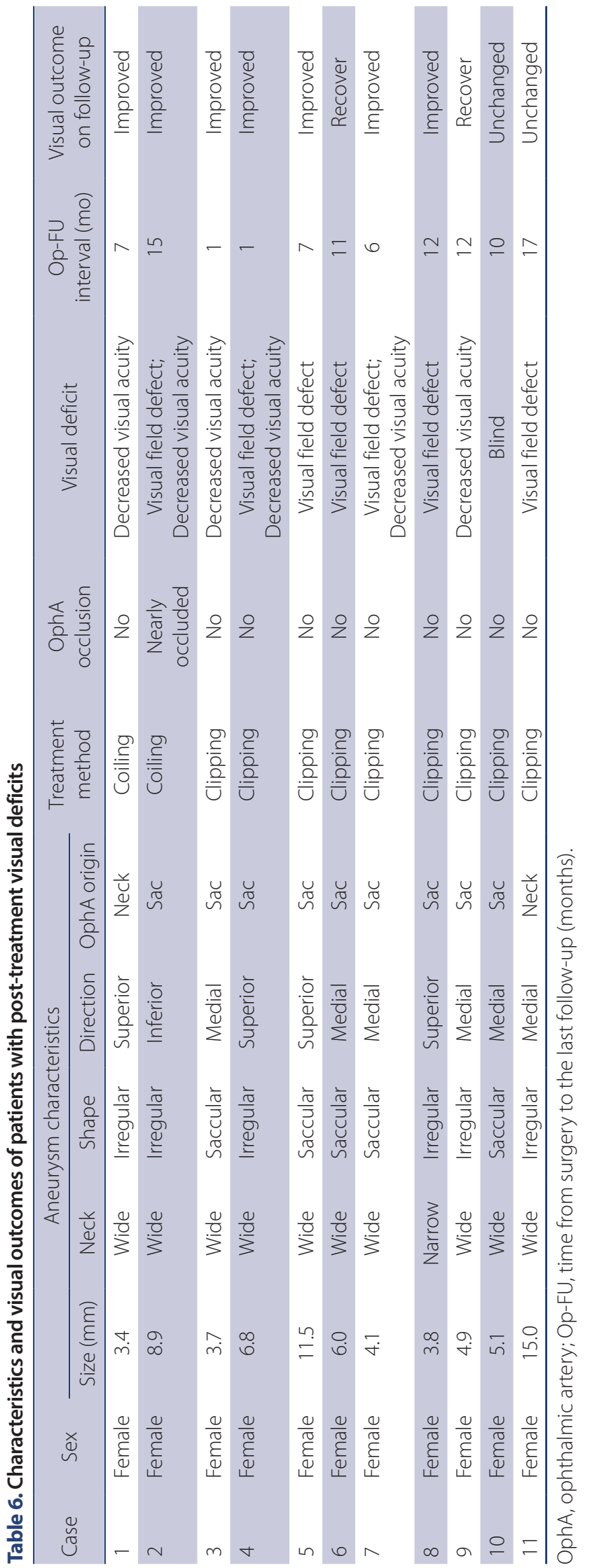

tions for OphA aneurysms treated by clipping is between $10.6 \%$ and $28.5 \%$, 2,11 while it is between 0 and $9.3 \%$ for patients treated by coiling. ${ }^{3,8,9,12}$ Our results are consistent with those of previous studies in which the incidence of newly appeared visual deficits was significantly higher in patients who underwent clipping than in those who underwent coiling ( $16.1 \%$ vs. $2.4 \%, P=0.010)$. Multivariate analysis also revealed that only microsurgical clipping resulted in a significant increase in the incidence of post-treatment visual deficits.

Kamide et al. ${ }^{2}$ analyzed 208 OphA aneurysms treated by microsurgical clipping and found that anterior clinoidectomy during surgery was associated with the incidence of new postoperative deficits. In our series, all 9 patients with newly acquired visual deficits had undergone anterior clinoidectomy during microsurgical clipping, and $89.3 \%$ of patients in the clipping group underwent anterior clinoidectomy, which was much higher than that in their study (75.3\%). This could be a possible explanation for the higher incidence of post-treatment visual deficits in our clipping group (16.1\% vs. 10.6\%). However, during the clipping of OphA aneurysms, anterior clinoidectomy is sometimes unavoidable because complete removal of this structure is required to expose the proximal neck of the aneurysm. Injury to the optic nerve during drilling of anterior clinoid process, ischemia of the optic pathway from perforator occlusion, or direct compression of the optic nerve by an applied clip may occur during this process, and all of these can cause visual deficits. ${ }^{2,13,14}$

In our series, 9 patients in the clipping group and 7 in the coiling group had pre-treatment visual deficits. Previous studies have indicated that microsurgical clipping is superior to endovascular treatment in the context of improvement or resolution of pre-treatment visual deficits, due to its ability to simultaneously eliminate compression and pulsation caused by the aneurysm. 13,15,16 However, in our series, the frequency of improvement of pre-treatment visual deficits did not differ between the 2 groups.

\section{Aneurysm Occlusion Outcomes}

A previous study has suggested that coiling of OphA aneurysms is associated with a high rate of incomplete aneurysm occlusion, which was $60.5 \%$ on angiography immediately after coiling. ${ }^{2,17}$ This could be due to attempts made by surgeons to save the OphA during the procedure, such as intentional partial coiling and avoidance of dense coil packing in the aneurysm. However, the incomplete aneurysm occlusion 
rate observed immediately or during follow-up in our series was comparable between the clipping and coiling groups. One possible reason for this is that the incomplete aneurysm occlusion rate in our coiling group was low as the BOT was performed to help treatment. This is substantiated by evidence indicating the lack of incomplete occlusion among patients with total or near OphA occlusion.

\section{Usefulness of the BOT for Coiling}

Previous studies have indicated that the BOT is helpful in predicting vision outcomes after coiling. Kim et al. ${ }^{8}$ reported that intact collateral circulation was demonstrated in 26 of 28 (92.9\%) patients who underwent the BOT in their series, and that no specific visual symptoms were observed in 3 patients with total occlusion of the OphA after coiling. Ahn et al. ${ }^{9}$ reported that an adequate collateral supply was identified in 12 of 14 (85.7\%) patients, and that there were no visual deficits in 4 patients with total OphA occlusion. Consistent with the results of these studies, we showed that intact collateral circulation was observed in $84.3 \%$ of patients, and there were no visual deficits in all 6 patients with total OphA occlusion. Therefore, we suggest that total occlusion of the OphA with an adequate collateral supply from the ECA, confirmed by the BOT, is safe during coiling for OphA aneurysms.

However, unpredicted thromboembolism events may occur during coiling for OphA aneurysms and can also cause post-treatment visual complications, ${ }^{4,5}$ which occurred in 2 patients in our coiling group, ie, 1 with good anterograde flow in the OphA and the other with significantly slow anterograde flow in the OphA. Retinal and/or optic ischemia could occur not only due to decreased perfusion of the completely occluded OphA, but also due to the variable degree of thromboembolic phenomenon from the orifice of the OphA during surgical or endovascular manipulation or the presence of any foreign material in the presence of any residual flow. As intentional total occlusion of the OphA is technically not easy with only coil packing, further studies are needed to determine whether partial occlusion of the OphA with an adequate collateral supply could increase the incidence of visual deficits.

The methods used to perform the BOT between studies are different. ${ }^{3,8,18}$ Undoubtedly, it is more precise to evaluate the collateral flow from ECA during the BOT if the OphA aneurysmal neck is occluded by a balloon catheter. However, this procedure also carries the risk of rupturing the aneurysm, and passing the balloon over the cavernous segment is fre- quently difficult. That is why we just put the balloon catheter at the petrous or cavernous segment of the ipsilateral ICA during BOT. Due to the presence of anterior and posterior communicating artery, the rate of real collateral circulation from ECA to OphA might be higher than $83.4 \%$ in the present study.

\section{Limitations}

There are several limitations to this study. First, this is a retrospective study, and there could be a selection bias in the choice of treatment modality. Second, the size of the aneurysms included in this study is relatively small, and no giant aneurysm was included, which could mask some risk factors associated with post-treatment visual complications. Third, we did not address the usefulness of flow diverters for the treatment of OphA aneurysms. In recent years, flow-diverter stents have emerged as a promising option in the management of intracranial aneurysms. Di Maria et al. ${ }^{19}$ conducted a comparative study between flow diverters and coiling for the treatment of carotid-ophthalmic aneurysms and found that aneurysms treated by flow diverters showed better long-term occlusion outcomes, though procedural complications and visual deficits seem higher in the patients treated by flow diverters. ${ }^{12,19}$ However, not all patients with OphA aneurysms could be treated with flow diverters because of the cost or regional policies, and therefore, our result that coiling is safer than clipping for the management of OphA aneurysms is still valuable.

\section{CONCLUSION}

In summary, our results revealed that compared with clipping, OphA aneurysms treated by coiling showed a comparable aneurysm occlusion rate and less post-treatment visual deficits. Careful consideration is necessary regarding the decision of surgical clipping for OphA aneurysms. Total occlusion of OphA with adequate collaterals during coiling did not cause visual deficits. However, further studies are needed to determine whether partial occlusion of the OphA with adequate collateral supply is associated with the incidence of thromboembolism events and visual deficits.

\section{Fund}

None. 


\section{Ethics Statement}

Data collection and analysis reported in this study were approved by the Institutional Review Board of our hospital. Informed consent for publication was not obtained so we anonymized the patient.

\section{Conflicts of Interest}

The authors have no conflicts to disclose.

\section{Author Contributions}

Concept and design: GL and DHL. Analysis and interpretation: GL, JC, JCP, JSA, and BDK. Data collection: GL, JC, JCP, JSA, and BDK. Writing the article: GL, JC, JCP, JSA, BDK, and DHL. Final approval of the article: GL, JCP, JSA, BDK, and DHL. Statistical analysis: GL. Overall responsibility: DHL.

\section{ORCID}

Guangdong Lu: https://orcid.org/0000-0003-3678-409X Jaewoo Chung: https://orcid.org/0000-0002-3512-6610 Jung Cheol Park: https://orcid.org/0000-0001-6677-455X Jae Sung Ahn: https://orcid.org/0000-0001-6134-6214 Byung Duk Kwun: https://orcid.org/0000-0001-7290-2087 Deok Hee Lee: https://orcid.org/0000-0003-0355-0449

\section{REFERENCES}

1. Kumon Y, Sakaki S, Kohno K, Ohta S, Ohue S, Oka Y. Asymptomatic, unruptured carotid-ophthalmic artery aneurysms: angiographical differentiation of each type, operative results, and indications. Surg Neurol 1997;48:465-472

2. Kamide T, Tabani H, Safaee MM, Burkhardt JK, Lawton MT. Microsurgical clipping of ophthalmic artery aneurysms: surgical results and visual outcomes with 208 aneurysms. J Neurosurg 2018;129:1511-1521

3. Yu JW, Shim YS, Lee JW, Kim DJ, Kim BM, Lim YC, et al. Vision outcomes of endovascular treatment for unruptured ophthalmic artery aneurysms. World Neurosurg 2018;116:e1223-e1229

4. Park H, Nakagawa I, Yokoyama S, Wada T, Motoyama Y, Kichikawa $\mathrm{K}$, et al. Central retinal artery thromboembolism without ophthalmic artery occlusion during stent-assisted coil embolization of ophthalmic artery aneurysm. World Neurosurg 2019;121:77-82

5. Elkordy AM, Sato K, Inoue Y, Mano Y, Matsumoto Y, Takahashi $A$, et al. Central retinal artery occlusion after the endovascular treatment of unruptured ophthalmic artery aneurysm: a case report and a literature review. NMC Case Rep J 2016;3:71-74

6. Castillo B Jr, De Alba F, Thornton J, DeBrun G, Pulido J. Retinal artery occlusion following coil embolization of carotid-ophthalmic aneurysms. Arch Ophthalmol 2000;118:851-852

7. Turner RD, Byrne JV, Kelly ME, Mitsos AP, Gonugunta V, Lalloo $S$, et al. Delayed visual deficits and monocular blindness after endovascular treatment of large and giant paraophthalmic aneurysms. Neurosurgery 2008;63:469-474; discussion 474-475

8. Kim B, Jeon P, Kim K, Yang N, Kim S, Kim H, et al. Endovascular treatment of unruptured ophthalmic artery aneurysms: clinical usefulness of the balloon occlusion test in predicting vision outcomes after coil embolization. J Neurointerv Surg 2016;8:696701

9. Ahn JH, Cho YD, Kang HS, Kim JE, Cho WS, Jung SC, et al. Endovascular treatment of ophthalmic artery aneurysms: assessing balloon test occlusion and preservation of vision in coil embolization. AJNR Am J Neuroradiol 2014;35:2146-2152

10. Horiuchi T, Yamamoto Y, Suzuki Y, Kobayashi M, Ichinose S, Hongo K. Clipping surgery for paraclinoid carotid aneurysm. Acta Neurochir Supp/ 2016;123:27-32

11. Matsukawa H, Tanikawa R, Kamiyama H, Tsuboi T, Noda K, Ota N, et al. Risk factors for visual impairments in patients with unruptured intradural paraclinoid aneurysms treated by neck clipping without bypass surgery. World Neurosurg 2016;91:183-189

12. Adeeb N, Griessenauer CJ, Foreman PM, Moore JM, Motiei-Langroudi R, Chua MH, et al. Comparison of stent-assisted coil embolization and the pipeline embolization device for endovascular treatment of ophthalmic segment aneurysms: a multicenter cohort study. World Neurosurg 2017;105:206-212

13. Date I, Asari S, Ohmoto T. Cerebral aneurysms causing visual symptoms: their features and surgical outcome. Clin Neurol Neurosurg 1998;100:259-267

14. Heran NS, Song JK, Kupersmith MJ, Niimi Y, Namba K, Langer DJ, et al. Large ophthalmic segment aneurysms with anterior optic pathway compression: assessment of anatomical and visual outcomes after endosaccular coil therapy. J Neurosurg 2007; 106:968-975

15. Tawk RG, Villalobos HJ, Levy El, Hopkins LN. Surgical decompression and coil removal for the recovery of vision after coiling and proximal occlusion of a clinoidal segment aneurysm: technical case report. Neurosurgery 2006;58:E1217; discussion E1217

16. Nanda A, Javalkar V. Microneurosurgical management of ophthalmic segment of the internal carotid artery aneurysms: single-surgeon operative experience from Louisiana State University, Shreveport. Neurosurgery 2011;68:355-370; discussion 370-371 
17. Shimizu K, Imamura H, Mineharu Y, Adachi H, Sakai C, Sakai N. Endovascular treatment of unruptured paraclinoid aneurysms: single-center experience with 400 cases and literature review. AJNR Am J Neuroradiol 2016;37:679-685

18. Sattur MG, Welz ME, Bendok BR, Miller JW. Balloon occlusion testing to assess retinal collateral and predict visual outcomes in the management of a fusiform intraorbital ophthalmic artery aneurysm: technical note and literature review. Oper Neurosurg (Hagerstown) 2019;16:60-66

19. Di Maria F, Pistocchi S, Clarençon F, Bartolini B, Blanc R, Biondi A, et al. Flow diversion versus standard endovascular techniques for the treatment of unruptured carotid-ophthalmic aneurysms. AJNR Am J Neuroradio/ 2015;36:2325-2330 